TRANSACTIONS OF THE

AMERICAN MATHEMATICAL SOCIETY

Volume 360, Number 11, November 2008, Pages 6103-6111

S 0002-9947(08)04481-4

Article electronically published on May 22, 2008

\title{
EVALUATING TAUTOLOGICAL CLASSES USING ONLY HURWITZ NUMBERS
}

\author{
AARON BERTRAM, RENZO CAVALIERI, AND GUEORGUI TODOROV
}

\begin{abstract}
Hurwitz numbers count ramified covers of a Riemann surface with prescribed monodromy. As such, they are purely combinatorial objects. Tautological classes, on the other hand, are distinguished classes in the intersection ring of the moduli spaces of Riemann surfaces of a given genus, and are thus "geometric". Localization computations in Gromov-Witten theory provide non-obvious relations between the two. This paper makes one such computation, and shows how it leads to a "master" relation (Theorem 0.1) that reduces the ratios of certain interesting tautological classes to the pure combinatorics of Hurwitz numbers. As a corollary, we obtain a purely combinatorial proof of a theorem of Bryan and Pandharipande, expressing in generating function form classical computations by Faber/Looijenga (Theorem 0.2).
\end{abstract}

\section{INTRODUCTION}

Clever applications of the Atiyah-Bott localization theorem in the context of Gromov-Witten theory have generated volumes of enumerative data as well as many insights into the structure of the tautological rings of the moduli spaces of curves (FP00, FP05, GJV01, GJV06). The general idea is to exploit torus actions on a target manifold (often just $\mathbb{P}^{1}$ ) to obtain torus actions on the moduli spaces of stable maps to the target. An analysis of the fixed loci for the torus action then produces intersection numbers and relations among tautological classes.

Recent work (GV01, GV03a, GV03b, Ion05]) has shown that the same idea, when applied to moduli of "relative" stable maps, reveals even more information. In this paper, we follow ideas of the second author in Cav06a, applying localization in the context of the ultimate relative stable map spaces to $\mathbb{P}^{1}$, the admissible cover spaces of [ACV01, to tie together enumerative data of apparently quite different natures by means of one succinct formula.

Theorem 0.1. For each fixed integer $d \geq 1$,

$$
\mathcal{C Y}(u)=\frac{(-1)^{d-1} d !}{d^{d}} \mathcal{H}_{(d)}(u) e^{d \mathcal{D}(u)},
$$

where

$\mathcal{H}_{(\mathbf{d})}(\mathbf{u})$ is the generating function for degree $d$, one-part simple Hurwitz numbers (properly defined in Section 1.1).

Received by the editors September 27, 2006 and, in revised form, January 20, 2007.

2000 Mathematics Subject Classification. Primary 14N35.

(C)2008 American Mathematical Society Reverts to public domain 28 years from publication 
$\mathcal{D}(\mathbf{u}) \quad$ is the generating function for $\lambda_{g} \lambda_{g-1}$ Hodge integrals on the moduli spaces $\mathcal{A}_{d d}^{g}$ (a.k.a. the evaluation of $\left[\mathcal{A}_{d d}^{g}\right]$ in $R^{g-2}\left(\mathcal{M}_{g}\right)$ )(discussed in the next paragraph and in Section 1.2).

$\mathcal{C Y}(\mathbf{u})$ is the generating function for the fully ramified Calabi-Yau cap invariants of degree d maps in Cav05b, briefly described in Section 1.3.

This new formula combines with other known formulas to explicitly reduce the enumerative invariants involved in the local Gromov-Witten theory of curves (BP04] to the "pure combinatorics" of single Hurwitz numbers.

One class of invariants appearing in Theorem 0.1 is worth singling out. The tautological ring $R^{*}\left(\mathcal{M}_{g}\right)$ of the moduli stack of curves of genus $g$ is the subring of the Chow ring (with rational coefficients) generated by Mumford's "kappa" classes (see Mum83). It is conjectured in Fab99 that this ring satisfies Poincaré duality with a (one-dimensional) socle in degree $g-2$. We focus on a particular family of degree $g-2$ tautological classes, which arise naturally from Hurwitz theory. Consider the $(2 g-1)$-dimensional "Hurwitz spaces":

$$
\mathcal{A}_{d d}^{g}:=\left\{\begin{aligned}
f: C \rightarrow \mathbb{P}^{1} \text { such that } \\
\\
\bullet C \text { is a connected curve of genus } g ; \\
\quad \bullet \text { is a map of degree } d ; \\
\quad f \text { is fully ramified over } 0 \text { and } \infty ; \\
\bullet f \text { is (at most) simply ramified over all other points }
\end{aligned}\right\} / P G L(2, \mathbb{C})
$$

and their induced source maps $s: \mathcal{A}_{d d}^{g} \rightarrow \mathcal{M}_{g}$ to the moduli space of curves (there is some sloppiness in this definition, as one needs to also factor by the automorphisms of $f)$. The Hurwitz spaces determine classes $\left[\mathcal{A}_{d d}^{g}\right] \in R^{g-2}\left(\mathcal{M}_{g}\right)$ that are multiples of a single class ( Loo95 $)$.

There is a smooth compactification $\mathcal{A}_{d d}^{g} \subset \overline{\mathcal{A}}_{d d}^{g}$ by the stack of admissible covers (Section 1.2) such that $s$ extends to a map $s: \overline{\mathcal{A}}_{d d}^{g} \rightarrow \overline{\mathcal{M}}_{g}$ to the Deligne-Mumford moduli stack of stable curves. A Hodge bundle $\mathbb{E}$ lives on $\overline{\mathcal{M}_{g}}$, whose Chern classes $\lambda_{1}, \ldots, \lambda_{g}$ are all tautological. Thus the following generating functions (if nonzero!) completely determine the proportionalities of the tautological classes $\left[\mathcal{A}_{d d}^{g}\right] \in$ $R^{*}\left(\mathcal{M}_{g}\right)$ :

$$
\mathcal{D}(u):=\sum_{g=1}^{\infty}\left(\int_{\overline{\mathcal{A}}_{d d}^{g}} \lambda_{g} \lambda_{g-1}\right) \frac{u^{2 g}}{2 g !} .
$$

Remark. It is not difficult to show that the class $\lambda_{g} \lambda_{g-1}$ vanishes on $\bar{A}_{d d}^{g} \backslash \mathcal{A}_{d d}^{g}$. This allows one to regard capping with $\lambda_{g} \lambda_{g-1}$ as an evaluation on the tautological ring $R^{*}\left(\mathcal{M}_{g}\right)$.

These generating functions were first computed by Bryan and Pandharipande in BP04, using Hodge integral techniques, a sophisticated geometric argument from Looijenga, and Faber's use of Mumford's computations in the hyperelliptic case $(d=2)$ that made use of the Grothendieck-Riemann-Roch theorem(!):

Theorem $0.2($ BP04, Thm 6.5).

$$
\mathcal{D}(u)=\ln \left(\frac{d \sin \left(\frac{u}{2}\right)}{\sin \left(\frac{d u}{2}\right)}\right) .
$$


Our point in this paper is to show how this (and other related) generating functions follow directly from localization on spaces of admissible covers. This reduces the computation to the combinatorics of simple Hurwitz numbers and, perhaps equally importantly, reduces the proof to a simple tallying up of fixed loci and their contributions to an equivariant integral. In other words, through localization, all the geometry has essentially been reduced to combinatorics.

\section{The Generating FunCtions}

This section serves the dual purpose of establishing our notation and generating function conventions, and introducing the main characters in the forthcoming computations. When dealing with families of enumerative invariants, it is very important to organize the information both naturally and efficiently. Our generating function conventions are driven both by geometry (in the choice of exponents of the formal parameters) and combinatorics (in the simple closed formulas for the generating functions with these conventions). This convention is already on display in the generating function $\mathcal{D}(u)$ of the introduction, where the exponent $u^{2 g}$ and denominator $2 g$ ! correspond to the number of simple ramification points $(2 g)$ of each of the maps $f: C \rightarrow \mathbb{P}^{1}$ of $\mathcal{A}_{d d}^{g}$.

1.1. Simple Hurwitz numbers. Simple Hurwitz numbers count ramified covers of the Riemann sphere with prescribed ramification data over one special point of $\mathbb{P}^{1}$ and simple ramification over the other (fixed) branch points. Let $\eta$ be a partition of the integer $d$. Then the associated simple Hurwitz number in genus $g$ is defined to be:

$$
H_{\eta}^{g}:=\sum_{\pi \in S_{\eta}^{g}} \frac{1}{|\operatorname{Aut}(\pi)|}
$$

where $S_{\eta}^{g}$ is the following set:

$$
S_{\eta}^{g}:=\left\{\begin{array}{l}
\text { degree } d \text { covers } \\
\text { such that: } C \stackrel{\pi}{\longrightarrow} \mathbb{P}^{1} \\
\bullet C \text { is a connected curve of genus } g ; \\
\bullet\left\{p_{1}, \ldots, p_{r}\right\} \text { are marked points in } \\
\text { fixed generic position on } \mathbb{P}^{1} ; \\
\bullet \pi \text { is unramified over } \\
\mathbb{P}^{1} \backslash\left\{\infty, p_{1}, \ldots, p_{r}\right\} ; \\
\bullet \pi \text { ramifies with profile } \eta \text { over } \infty ; \\
\bullet \pi \text { has simple ramification over } p_{i} ;
\end{array}\right\}
$$

and $\operatorname{Aut}(\pi)$ is the (finite!) automorphism group of each such map $\pi$.

Remark. The number $r$ of simple branch points is determined by the RiemannHurwitz formula:

$$
r=2 g+d-2+\ell(\eta)
$$

where $\ell(\eta)$ is the length of the partition $\eta$.

Using this as a definition for $r$, we organize Hurwitz numbers in generating function form as follows:

$$
\mathcal{H}_{\eta}(u):=\sum_{g=0}^{\infty}(-1)^{g} H_{\eta}^{g} \frac{u^{r}}{r !} .
$$


Remark. The sign $(-1)^{g}$ is non-standard, and has no particular meaning but to tune this generating function to our localization set-up.

For the $d$-cycle $\eta=(d)$, the generating function $\mathcal{H}_{(d)}(u)$ has a particularly simple form that was first computed by Shapiro, Shapiro and Vainshtein in [SSV97]:

Formula 1.1 ( SSV97, Thm 6).

$$
\mathcal{H}_{(d)}(u)=\frac{2^{d-1}}{d \cdot d !}\left(\sin \left(\frac{d u}{2}\right)\right)^{d-1}
$$

In general, the single Hurwitz numbers are harder to compute. However, the following relation among single Hurwitz numbers was obtained by localization techniques by the second author in Cav05b:

Formula 1.2 (Cav05b], Equation (26)).

$$
0=\sum_{\eta \vdash d}(-1)^{\ell(\eta)} \frac{\mathcal{H}_{\eta}(u)}{\prod_{\eta_{i} \in \eta} 2 \sin \left(\frac{\eta_{i} u}{2}\right)}
$$

where $\eta_{i} \in \eta$ are the cycle-lengths of the partition $\eta$.

1.2. $\lambda_{g} \lambda_{g-1}$ Hurwitz-Hodge integrals. Let:

$$
\overline{\mathcal{A}}_{d d}^{g}=\overline{A d m}_{g \stackrel{d}{\rightarrow} 0,\left(t_{1}, \ldots, t_{2 g},(d)_{2 g+1},(d)_{2 g+2}\right)}
$$

be the Abramovich-Corti-Vistoli (smooth, proper) moduli stack (ACV01]) of:

- genus $g$, degree $d$ admissible covers of a genus 0 (possibly nodal) curve;

- with all $2 g+2$ branch points marked;

- the first $2 g$ branch points carrying a simple ramification profile;

- the last two branch points carrying ramification profile $(d)$.

The universal family of admissible covers defines source and target maps:

$$
\begin{gathered}
\overline{\mathcal{A}}_{d d}^{g} \quad \stackrel{s}{\rightarrow} \overline{\mathcal{M}}_{g, 2} \\
\overline{\mathcal{M}}_{0,2 g+2}
\end{gathered}
$$

to the moduli spaces of genus $g$ stable curves with two marked points (corresponding to the fully ramified points) and of genus $0,2 g+2$-pointed stable curves.

Let $\lambda_{i}$ be the Chern classes of the Hodge bundle (pulled back from $\overline{\mathcal{M}}_{g, 2}$ ), and, in addition, let $\psi=t^{*} \psi_{2 g+2}$ be the pull-back of the Mumford-Morita class from $\overline{\mathcal{M}}_{0,2 g+2}$ (Mum83). Integrals of products of such classes are referred to as Hurwitz-Hodge integrals ([BGP05]) and following [Cav06a], we define the following generating functions of Hurwitz-Hodge integrals:

$$
\mathcal{D}_{i}(u):=\sum_{g \geq i}\left(\int_{\bar{A}_{d d}^{g}} \lambda_{g} \lambda_{g-i} \psi^{i-1}\right) \frac{u^{2 g}}{2 g !} .
$$

In particular, $\mathcal{D}(u)=\mathcal{D}_{1}(u)$. 
These generating functions are all related via:

Formula 1.3 ( Cav06a, Thm 1).

$$
\mathcal{D}_{i}(u):=\frac{d^{i-1}}{i !}(\mathcal{D}(u))^{i}
$$

Remark. Thus if we set $\mathcal{D}_{0}(u):=\frac{1}{d}$, then we may combine the $\mathcal{D}_{i}(u)$ in:

$$
\mathcal{T}(u)=\sum_{g \geq 0} T^{g} \frac{u^{2 g}}{2 g !}:=\sum_{i=0}^{\infty} \mathcal{D}_{i}(u)=\frac{1}{d} \mathrm{e}^{d \mathcal{D}(u)} .
$$

1.3. CY cap. Consider the integral:

$$
C Y^{g}:=\int_{\star} c_{g}\left(\mathbb{E}^{*}\right) c_{g+d-1}\left(R^{1} \pi_{*} f^{*}\left(\mathcal{O}_{\mathbb{P}^{1}}(-1)\right)\right) e v_{(d)}^{*}(\infty),
$$

where:

$$
\star:=\overline{A d m}_{g \stackrel{d}{\rightarrow} \mathbb{P}^{1},\left((d), t_{1}, \ldots, t_{2 g+d-1}\right)}
$$

is the moduli space of degree $d$, genus $g$ admissible covers of a parametrized $\mathbb{P}^{1}$, fully ramified over one special branch point, simple ramification over all other (marked!) branch points (see Cav05a, section 1.1.6.2);

- $\pi: \mathcal{U} \rightarrow \star$ is the projection from the universal admissible cover family;

- $f: \mathcal{U} \rightarrow \mathbb{P}^{1}$ is the universal map 1 ;

- $e v_{(d)}: \star \rightarrow \mathbb{P}^{1}$ is the map evaluating a cover at its unique fully ramified point (i.e. the composition of $f$ with the first section $s_{(d)}: \star \rightarrow \mathcal{U}$ ).

Define the generating function:

$$
\mathcal{C Y}(u):=\sum_{g=0}^{\infty} C Y^{g} \frac{u^{2 g+d-1}}{(2 g+d-1) !} .
$$

Localization and relations among simple Hurwitz numbers determine this generating function. The computation is carried out in Cav06b for degrees 2,3 and in Cav05b for general degree. The following result is obtained by localizing and reducing to Formula 1.2 above:

Formula 1.4 ( Cav05b, Thm 3).

$$
\mathcal{C Y}(u)=(-1)^{d-1} \frac{1}{d} \frac{\left(2 \sin \left(\frac{u}{2}\right)\right)^{d}}{2 \sin \left(\frac{d u}{2}\right)}
$$

Remarks.

(1) The generating function $C Y$ is a fundamental quantity in the TQFT of Admissible Cover invariants (Cav05b): it represents the only non-zero connected invariants for the Calabi-Yau cap, i.e. the one-pointed invariants for the zero section of the bundle $\mathcal{O}_{\mathbb{P}^{1}} \oplus \mathcal{O}_{\mathbb{P}^{1}}(-1)$. It is also a close relative of the analogous invariants in the local GW theory of curves ([BP04]).

\footnotetext{
${ }^{1}$ Followed by contraction of the "sprouted twigs".
} 
This explains its otherwise slightly mysterious presence (and name) in this application.

(2) There is one small notational difference between the description of $\mathcal{C Y}(u)$ here and in Cav05b. There, we worked on moduli spaces of covers where the branch locus on $\mathbb{P}^{1}$ was not marked. Here, we mark all branch points on $\mathbb{P}^{1}$, and then include the "normalization factor" $1 /(2 g+d-1)$ ! to compensate. The two choices are obviously equivalent.

\section{Proof of theorems}

\subsection{Localization computation.}

2.1.1. The set-up. Consider the one-dimensional algebraic torus $\mathbb{C}^{*}$, and recall that the $\mathbb{C}^{*}$-equivariant Chow ring of a point is a polynomial ring in one variable:

$$
A_{\mathbb{C}^{*}}^{*}(\{p t\}, \mathbb{C})=\mathbb{C}[\hbar] .
$$

Let $\mathbb{C}^{*}$ act on a two-dimensional vector space $V$ via:

$$
t \cdot\left(z_{0}, z_{1}\right)=\left(t z_{0}, z_{1}\right) .
$$

This action descends to $\mathbb{P}^{1}$, with fixed points $0=(1: 0)$ and $\infty=(0: 1)$. An equivariant lifting of $\mathbb{C}^{*}$ to a line bundle $L$ over $\mathbb{P}^{1}$ is uniquely determined by its weights $\left\{L_{0}, L_{\infty}\right\}$ over the fixed points.

The action on $\mathbb{P}^{1}$ induces an action on the moduli spaces of admissible covers to a parametrized $\mathbb{P}^{1}$ simply by post composing the cover map with the automorphism of $\mathbb{P}^{1}$ defined by $t$.

The fixed loci for the induced action on the moduli space consist of admissible covers such that anything "interesting" (ramification, nodes, marked points) happens over 0 or $\infty$, or on "non-special" twigs that attach to the main $\mathbb{P}^{1}$ at 0 or $\infty$.

In the following treatment we use the "localization language" and notations in $\mathrm{HKK}^{+} 03$, chapters 4 and 27. More extensive discussions of the application of localization in the context of admissible covers are in Cav06b] and Cav05a].

2.1.2. The strategy. In [Cav05b], the second author proved Formula 1.4 using localization. The integrand in (5) can be regarded as an equivariant cohomology class. A well chosen linearization for the bundles $\mathcal{O}_{\mathbb{P}^{1}}$ and $\mathcal{O}_{\mathbb{P}^{1}}(-1)$ allows the explicit evaluation of it - which is a non-equivariant quantity. Hence, such evaluation is independent of the linearization chosen. Here we use a different system of linearization weights to evaluate (5):

\begin{tabular}{|l||c|c|}
\hline & weight over 0 & weight over $\infty$ \\
\hline \hline $\mathcal{O}_{\mathbb{P}^{1}}(-1)$ & -1 & 0 \\
\hline $\mathcal{O}_{\mathbb{P}^{1}}$ & 1 & 1 \\
\hline
\end{tabular}

2.1.3. The possibly contributing fixed loci. The above choice of linearization restricts the number of possibly contributing fixed loci (identified here with their localization graphs):

(1) The full ramification condition at $\infty$ forces the preimages of $\infty$ to be connected.

(2) The weight " 0 " over $\infty$ forces the localization graphs to have valence 1 over $\infty$ (see, e.g., Cav06b], section 2, or BP05] for a very similar discussion in the setting of stable maps). 

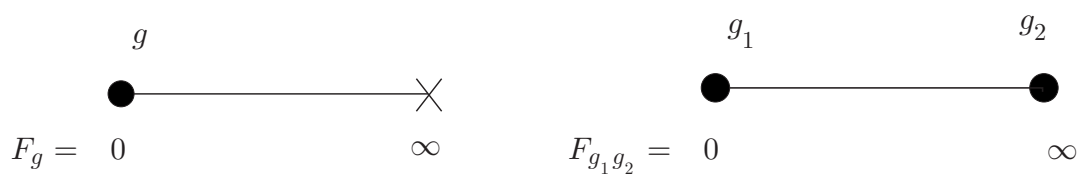

Figure 1. The localization graphs of the possibly contributing fixed loci.

The possibly contributing fixed loci consist of boundary strata parameterizing a single sphere, fully ramified over 0 and $\infty$, mapping with degree $d$ to the main $\mathbb{P}^{1}$. A rational tail $T_{0}$ must sprout from 0 , and be covered by a curve of genus $g_{1}$, $0 \leq g_{1} \leq g$. If $g_{1}<g$, then a rational tail $T_{\infty}$ sprouts from $\infty$ as well, covered by a curve $C$ of genus $g_{2}=g-g_{1}$. The map $C \rightarrow T_{\infty}$ has two points of full ramification: the attaching point and another point, corresponding to the original mark $(d)$. The situation is illustrated in Figure 1 .

These fixed loci are covers of products of spaces of admissible covers, with "stacky" multiplicities (see Cav05a for a quick explanation, or AGV06 for a comprehensive treatise of this subject):

$$
F_{g} \cong \overline{\mathcal{A}_{d}^{g}}, \quad F_{g_{1} g_{2}} \rightarrow \overline{\mathcal{A}}_{d}^{g_{1}} \times \overline{\mathcal{A}}_{d d}^{g_{2}} \text { of degree } d\left(\begin{array}{c}
2 g+d-1 \\
2 g_{2}
\end{array}\right),
$$

with the following notation:

$$
\begin{aligned}
& \text { - } \overline{\mathcal{A}}_{d}^{g}:=\overline{A d m}_{g \stackrel{d}{a} 0,\left(t_{1}, \ldots, t_{2 g+d-1},(d)\right)} ; \\
& \text { - } \overline{\mathcal{A}}_{d d}^{g}:=\overline{A d m}_{g \stackrel{d}{\rightarrow} 0,\left(t_{1}, \ldots, t_{2 g},(d)_{2 g+1},(d)_{2 g+2}\right)} .
\end{aligned}
$$

2.1.4. The relations. Before we write down the explicit contributions to integral (5), let us introduce one last piece of notation that is intended to make the computation a little less painful to read:

$$
\Lambda_{g}(n):=\sum_{i=0}^{g}(n \hbar)^{i} \lambda_{g-i}
$$

Remark 1. It is useful to interpret in this notation Mumford's relation (Mum83]) about Chern classes of the Hodge bundle: $c_{t}\left(\mathbb{E} \oplus \mathbb{E}^{*}\right)=c_{t}(\mathbb{E}) c_{t}\left(\mathbb{E}^{*}\right)=1$.

Since

- $\Lambda_{g}(1):=\sum_{i=0}^{g}(\hbar)^{i} \lambda_{g-i}=\hbar^{g} c_{\frac{1}{\hbar}}(\mathbb{E})$,

- $\Lambda_{g}(-1):=\sum_{i=0}^{g}(-\hbar)^{i} \lambda_{g-i}=(-\hbar)^{g} c_{\frac{1}{\hbar}}\left(\mathbb{E}^{*}\right)$,

it follows that

$$
\Lambda_{g}(1) \Lambda_{g}(-1)=(-1)^{g} \hbar^{2 g} .
$$

Remark 2. We deduce the identity

$$
\int_{\overline{\mathcal{A}}_{d}^{g}} \psi^{2 g+d-3}=H_{(d)}^{g}
$$

( $\psi$ is defined on $\overline{\mathcal{A}}_{d}^{g}$ as it is done in Section 1.2 for $\overline{\mathcal{A}}_{d d}^{g}$ ) from the two facts:

- $H_{(d)}^{g}$ is the degree of the map $\overline{\mathcal{A}}_{d}^{g} \rightarrow \overline{\mathcal{M}}_{0,2 g+d}$;

- $\int \overline{\mathcal{M}}_{0,2 g+d} \psi^{2 g+d-3}=1$. 
With the choice of linearization from Section 2.1.2 the contribution to $C Y^{g}$ from the fixed locus $F_{g_{1} g_{2}}$ is:

$$
d\left(\begin{array}{c}
2 g+d-1 \\
2 g_{2}
\end{array}\right)(-\hbar)^{d-1} \frac{(d-1) !}{d^{d-1}} \int_{\overline{\mathcal{A}}_{d}^{g_{1}}} \frac{\Lambda_{g_{1}}(-1) \Lambda_{g_{1}}(+1)}{\hbar(\hbar-\psi)} \int_{\overline{\mathcal{A}}_{d d}^{g_{2}}} \frac{\Lambda_{g_{2}}(-1) \lambda_{g_{2}}}{\hbar(\hbar+\psi)} .
$$

Expanding the denominators and using (7) this reduces to:

$$
\begin{array}{r}
\frac{(-1)^{d-1} d !}{d^{d-1}}\left(\begin{array}{c}
2 g+d-1 \\
2 g_{2}
\end{array}\right) \int_{\overline{\mathcal{A}}_{d}^{g_{1}}}(-1)^{g_{1}} \psi^{2 g_{1}+d-3} \int_{\overline{\mathcal{A}}_{d d}^{g_{2}}} \lambda_{g_{2}} \lambda_{g_{2}-1}+\ldots+\lambda_{g_{2}} \psi^{g_{2}-1} \\
=\frac{(-1)^{d-1} d !}{d^{d-1}}(2 g+d-1) ! H_{(d)}^{g_{1}} T^{g_{2}} .
\end{array}
$$

The contribution of $F_{g}$ is:

$$
(-\hbar)^{d-1} \frac{(d-1) !}{d^{d-1}} \int_{\overline{\mathcal{A}}_{d}^{g}} \frac{\Lambda_{g}(-1) \Lambda_{g}(+1)}{\hbar(\hbar-\psi)}=\frac{(-1)^{d-1}(d-1) !}{d^{d-1}}(2 g+d-1) ! H_{(d)}^{g},
$$

which, since $T^{0}=\mathcal{D}_{0}=1 / d$, can be rewritten as

$$
\frac{(-1)^{d-1} d !}{d^{d-1}}(2 g+d-1) ! H_{(d)}^{g} T^{0}
$$

Equations (9) and (10) are nicely encoded in generating function form to give the relation:

$$
\mathcal{C Y}(u)=\frac{(-1)^{d-1} d !}{d^{d-1}} \mathcal{H}_{(d)}(u) \mathcal{T}(u) .
$$

Theorem 0.1 follows immediately from (11) and (4).

Theorem 0.2 is a straightforward corollary of Theorem 0.1 , recall that the combinatorics of simple Hurwitz numbers gives us explicit expressions for $\mathcal{H}_{(d)}(u)$ (Formula 1.1) and $\mathcal{C} \mathcal{Y}(u)$ (Formula 1.4). Solving for $\mathcal{D}(u)$ we obtain:

$$
\mathcal{D}(u)=\ln \left(\frac{d \sin \left(\frac{u}{2}\right)}{\sin \left(\frac{d u}{2}\right)}\right) .
$$

\section{ACKNOWLEDGEMENT}

Some suspicious-looking similarities among generating functions in local GromovWitten theory prompted Ravi Vakil to demand an explanation of the second author. This paper (we hope) serves as an explanation.

\section{REFERENCES}

[ACV01] Dan Abramovich, Alessio Corti, and Angelo Vistoli. Twisted bundles and admissible covers. Comm in Algebra, 31(8):3547-3618, 2001. MR2007376 (2005b:14049)

[AGV06] Dan Abramovich, Tom Graber, and Angelo Vistoli. Gromov-Witten theory of DeligneMumford stacks. Preprint: math.AG/0603151, 2006.

[BGP05] Jim Bryan, Tom Graber, and Rahul Pandharipande. The orbifold quantum cohomology of $\mathbb{C}^{2} / \mathbb{Z}_{3}$ and Hurwitz-Hodge integrals. Preprint:math.AG/0510335, 2005.

[BP04] Jim Bryan and Rahul Pandharipande. The local Gromov-Witten theory of curves. Preprint: math.AG/0411037, 2004.

[BP05] Jim Bryan and Rahul Pandharipande. Curves in Calabi-Yau threefolds and topological quantum field theory. Duke Math. J., 126(2):369-396, 2005. MR2115262 (2005k:14117) 
[Cav05a] Renzo Cavalieri. Phd thesis. http://www.math.lsa.umich.edu/ crenzo/ thesis.pdf, 2005.

[Cav05b] Renzo Cavalieri. A TQFT for intersection numbers on moduli spaces of admissible covers. Preprint: mathAG/0512225, 2005.

[Cav06a] Renzo Cavalieri. Generating functions for Hurwitz-Hodge integrals. Preprint:mathAG/0608590, 2006.

[Cav06b] Renzo Cavalieri. Hodge-type integrals on moduli spaces of admissible covers. In Dave Auckly and Jim Bryan, editors, The interaction of finite type and Gromov-Witten invariants (BIRS 2003), volume 8. Geometry and Topology monographs, 2006.

[Fab99] Carel Faber. A conjectural description of the tautological ring of the moduli space of curves. In Moduli of curves and abelian varieties, Aspects Math., E33, pages 109-129. Vieweg, Braunschweig, 1999. MR.1722541 (2000j:14044)

[FP00] Carel Faber and Rahul Pandharipande. Hodge integrals and Gromov-Witten theory. Invent. Math., 139(1):173-199, 2000. MR.1728879 (2000m:14057)

[FP05] C. Faber and R. Pandharipande. Relative maps and tautological classes. J. Eur. Math. Soc. (JEMS), 7(1):13-49, 2005. MR2120989 (2005m:14046)

[GJV01] I. P. Goulden, D. M. Jackson, and R. Vakil. The Gromov-Witten potential of a point, Hurwitz numbers, and Hodge integrals. Proc. London Math. Soc. (3), 83(3):563-581, 2001. MR1851082 (2002h:14043)

[GJV06] Ian Goulden, David Jackson, and Ravi Vakil. A short proof of the $\lambda_{g}$-conjecture without Gromov-Witten theory: Hurwitz theory and the moduli of curves. Preprint:mathAG/0604297, 2006.

[GV01] Tom Graber and Ravi Vakil. On the tautological ring of $\bar{M}_{g, n}$. Turkish J. Math., 25(1):237-243, 2001. MR1829089 (2002b:14034)

[GV03a] Tom Graber and Ravi Vakil. Hodge integrals, Hurwitz numbers, and virtual localization. Compositio Math., 135:25-36, 2003. MR1955162 (2004c:14108)

[GV03b] Tom Graber and Ravi Vakil. Relative virtual localization and vanishing of tautological classes on moduli spaces of curves. Duke Math J., 130(1):1-37, 2005. MR2176546 (2006j:14035)

$\left[\mathrm{HKK}^{+} 03\right]$ Kentaro Hori, Sheldon Katz, Albrecht Klemm, Rahul Pandharipande, Richard Thomas, Cumrun Vafa, Ravi Vakil, and Eric Zaslow. Mirror Symmetry. AMS CMI, 2003. MR2003030 (2004g:14042)

[Ion05] Eleny-Nicoleta Ionel. Relations in the tautological ring of $M_{g}$. Duke Math. J., 129(1):157-186, 2005. MR2155060 (2006c:14040)

[Loo95] Eduard Looijenga. On the tautological ring of $M_{g}$. Invent. Math., 121(2):411-419, 1995. MR1346214 (96g:14021)

[Mum83] David Mumford. Toward an enumerative geometry of the moduli space of curves. Arithmetic and Geometry, II(36):271-326, 1983. MR717614 (85j:14046)

[SSV97] B. Shapiro, M. Shapiro, and A. Vainshtein. Ramified coverings of $S^{2}$ with one degenerate branch point and enumeration of edge-ordered graphs. In Topics in singularity theory, volume 180 of Amer. Math. Soc. Transl. Ser. 2, pages 219-227. Amer. Math. Soc., Providence, RI, 1997. MR1767127 (2002d:57001)

Department of Mathematics, University of Utah, 155 South 1400 East, Rm 233, Salt Lake City, Utah 84112-0090

Department of Mathematics, University of Michigan, 2074 East Hall, 530 Church Street, Ann Arbor, Michigan 48109-1043

Department of Mathematics, University of Utah, Salt Lake City, Utah 84112-0090 